\title{
EINE FRÜHNEUZEITLICHE „FAMILIENUNIVERSITÄT“"? DAS PROFESSORENKOLLEGIUM DER NÜRNBERGER HOHEN SCHULE UND UNIVERSITÄT ALTDORF 1575-1809
}

\author{
WOLFGANG MÄHRLE
}

\section{AN EARLY-MODERN 'FAMILY UNIVERSITY'? THE PROFESSORIAL BODY OF THE NUREMBERG ACADEMY AND UNIVERSITY IN ALTDORF 1575-1809}

The article applies methods of collective biography to examine the professorial body at the academy or (since 1623) university in Altdorf between the years 1575 and 1809. The subject of the analysis is the geographical and social origin of full-time professors, their pursuit of education, and their life careers, including the execution of offices. In closing, the article takes a position on the question of whether or not Altdorf can be called a 'family university'.

Keywords: Altdorf - Nuremberg - professorial body - scholarship - family university - family of scholars - prosopography - recruitment

DOI: $10.14712 / 23365730.2020 .17$

Die Professorenkollegien frühneuzeitlicher Universitäten als sozialhistorische Formationen haben in der jüngeren Vergangenheit verstärkt das Interesse der Forschung gefunden. ${ }^{1}$ Inzwischen liegen über das Lehrpersonal einer ganzen Reihe von mitteleuropäischen Hochschulen Kollektivbiografien vor. ${ }^{2}$ Diese Studien tragen erheblich zum Verständnis sowohl

1 Forschungsbericht zur Universitätsgeschichtsschreibung: Matthias AschE - Stefan GERBER, Neuzeitliche Universitätsgeschichte in Deutschland. Entwicklungslinien und Forschungsfelder, Archiv für Kulturgeschichte 90, 2008, S. 159-201.

2 Vgl. bes. Hermann Niebuhr, Zur Sozialgeschichte der Marburger Professoren 1653-1806, Darmstadt-Marburg 1983; Karl Henning Wolf, Die Heidelberger Universitätsangehörigen im 18. Jahrhundert. Studien zu Herkunft, Werdegang und sozialem Beziehungsgeflecht, Heidelberg 1991, hier S. 26-51; Bernhard ZASCHKA, Die Lehrstühle der Universität Tübingen im Dreißigjährigen Krieg. Zur sozialen Wirklichkeit von Professoren im vorklassischen Zeitalter, Tübingen 1993; Trude MAURER, Hochschullehrer im Zarenreich. Ein Beitrag zur russischen Sozial- und Bildungsgeschichte, Köln-Weimar-Wien 1998 (hier bes. auch der Abschnitt zur Methodik kollektivbiografischer Studien, S. 10-23); Olaf WiLlett, Sozialgeschichte Erlanger Professoren 1743-1933, Göttingen 2001; DERS., Biographischer Apparat zur Sozialgeschichte Erlanger Professoren 1743-1933, Erlangen 2001; Bernhart JäHNIG, Die Königsberger Universitätsprofessoren im 17. Jahrhundert. Eine sozial- und bildungsgeschichtliche Betrachtung, in: Klaus Garber - Manfred Komorowski - Axel E. Walter (Hgg.), Kulturgeschichte Ostpreußens in der Frühen Neuzeit, Tübingen 2001, S. 337-373; Nils JöRN, Die Herkunft der Professorenschaft der Greifswalder Universität zwischen 1456 und 1815, in: Dirk Alvermann ders. - Jens E. Olesen (Hgg.), Die Universität Greifswald in der Bildungslandschaft des Ostseeraums, Berlin 2007, S. 155-190; Matthias Asche, Helmstedter Professorenprofile 1576-1810. Skizzen zur Kollektivbiographie einer mitteldeutschen Universität, in: Jens Bruning - Ulrike Gleixner (Hgg.), Das Athen der Welfen. Die Reformuniversität Helmstedt 1576-1810, Wiesbaden 2010, S. 114-119; DERs., Biographische Profile und Rekrutierungsmechanismen von Professoren an kleinen und mittelgrossen protestantischen Universitäten im 
institutionen- als auch wissenschaftsgeschichtlicher Entwicklungen an den untersuchten Bildungseinrichtungen bei. So werden beispielsweise an vielen Universitäten die spezifischen Rekrutierungsmuster von Professoren erst durch die Kenntnis sozialhistorischer Zusammenhänge verständlich. Eine zu gruppenbiografischen Forschungen komplementäre Perspektive auf die Professorenkollegien frühneuzeitlicher Hochschulen eröffnen Studien über Gelehrtenfamilien, wie beispielsweise die Dissertation von Julian Kümmerle über die württembergische Familie Bidembach. ${ }^{3}$

Die Nürnberger Hohe Schule und Universität Altdorf fand im Kontext der neueren Forschungen über die Professorenkollegien der Vormoderne bisher keine Beachtung. Dies liegt nicht zuletzt daran, dass für die Altorfina kein moderner Professorenkatalog vorliegt, auf dessen personengeschichtlichen Informationen entsprechende Forschungen aufbauen könnten. Analysen zu Altdorf müssen sich in erster Linie auf die Angaben frühneuzeitlicher Gelehrtenlexika stützen, vor allem auf die Einträge im „Nürnbergischen Gelehrten-Lexicon", das von Georg Andreas Will und Christian Conrad Nopitsch in den Jahren 1755-1758 bzw. 1802-1808 in acht Bänden herausgegeben wurde. ${ }^{4}$

Im Folgenden untersuche ich das Professorenkollegium der Altdorfer Hochschule von 1575 bis 1809 unter kollektivbiografischen Fragestellungen. Ich beziehe also in meine Analysen sowohl die Zeit des akademischen Gymnasiums bzw. der „Semiuniversitas“ bis 1623

Heiligen Römischen Reich 1650-1800. Eine prosopographisch-kollektivbiographische Analyse von Professorenlexika, in: Christian Hesse - Rainer Christoph Schwinges (Hgg.), Professorinnen und Professoren gewinnen. Zur Geschichte des Berufungswesens an den Universitäten Mitteleuropas, Basel 2012, S. 185-245; Theresa Scнмотz, Die Leipziger Professorenfamilien im 17. und 18 Jahrhundert. Eine Studie über Herkunft, Vernetzung und Alltagsleben, Leipzig-Stuttgart 2012; Julia ScHOPFERER, Sozialgeschichte der halleschen Professoren 1694-1806. Lebenswege, Netzwerke und Raum als Strukturbedingungen von universitärer Wissenschaft und frühmoderner Gelehrtenexistenz, Halle (Saale) 2016; Bernhard Homa, Die Tübinger Philosophische Fakultät 1652-1752. Institutionen - Disziplinen - Lehrkräfte, Stuttgart 2016; Swantje PioTrowsKi, Sozialgeschichte der Kieler Professorenschaft 1665-1815. Gelehrtenbiographien im Spannungsfeld zwischen wissenschaftlicher Qualifikation und sozialen Verflechtungen, Kiel-Hamburg 2018.

3 Julian KüMmerLe, Luthertum, humanistische Bildung und württembergischer Territorialstaat. Die Gelehrtenfamilie Bidembach vom 16. bis zum 18. Jahrhundert, Stuttgart 2008. Grundlegend zu mitteleuropäischen Gelehrtenfamilien: Friedrich Wilhelm EuLER, Entstehung und Entwicklung deutscher Gelehrtengeschlechter, in: Hellmuth Rössler - Günther Franz (Hgg.), Universität und Gelehrtenstand 1400-1800, Limburg/Lahn 1970, S. 183-232; Julian KüMmERLE, Wissenschaft und Verwandtschaft. Protestantische Theologenausbildung im Zeichen der Familie vom 16. bis zum 18. Jahrhundert, in: Herman Selderhuis - Markus Wriedt (Hgg.), Bildung und Konfession. Theologenausbildung im Zeitalter der Konfessionalisierung, Tübingen 2006, S. 159-210; DERS., Konfessionalität und Gelehrtenkultur im Generationenverband. Protestantische Theologen- und Juristenfamilien im Alten Reich, in Frankreich und der Schweiz, in: Thomas Kaufmann - Anselm Schubert - Kaspar von Greyerz (Hgg.), Frühneuzeitliche Konfessionskulturen, Göttingen 2008, S. 69-97. Zur zeitweise in Nürnberg ansässigen Gelehrtenfamilie Camerarius vgl. Gerhard SeIBoLD, Die Cammermeister genannt CamerariiBeamte, Kaufleute, Wissenschaftler, Politiker, Jahrbuch für fränkische Landesforschung 67, 2007, S. 107-160; Wolfgang MährLe, Bildungspolitik im Zeichen Melanchthons. Die Familie Camerarius und das höhere Schulwesen in Nürnberg 1526-1624, in: Hanspeter Marti - Karin Marti-Weissenbach (Hgg.), Nürnbergs Hochschule in Altdorf. Beiträge zur frühneuzeitlichen Wissenschafts- und Bildungsgeschichte, Köln-Weimar-Wien 2014, S. 17-40.

4 Georg Andreas WiLL, Nürnbergisches Gelehrten-Lexicon oder Beschreibung aller Nürnbergischen Gelehrten beyderley Geschlechtes nach Ihrem Leben, Verdiensten und Schrifften [...], I-IV, Nürnberg-Altdorf 1755-1758; fortgesetzt von Christian Conrad NopITSCH V-VIII, Altdorf 1802-1808. Die wichtigsten biografischen Daten zu den Altdorfer Professoren enthält eine von Werner Wilhelm Schnabel herausgegebene DVD, vgl. Werner Wilhelm Schnabel (Hg.), Athena Norica. Bilder und Daten zur Geschichte der Universität Altdorf [DVD-ROM], Nürnberg 2012. 
als auch die anschließende Universitätszeit ein. ${ }^{5}$ Den Endpunkt des Untersuchungszeitraums markiert die Auflösung der Altdorfer Universität im Jahr 1809, wenige Jahre nach dem Übergang der Reichsstadt Nürnberg an Bayern.

Die Academia Norica zählte seit dem ausgehenden 16. Jahrhundert zu den mittelgroßen, in den letzten Jahrzehnten ihres Bestehens zu den kleinen Universitäten im Heiligen Römischen Reich. Der Bedeutungsverlust der Altdorfer Hochschule als Bildungseinrichtung spiegelt sich in der Entwicklung der Immatrikulationen. ${ }^{6}$ Die Zahl der Studenten in Altdorf erreichte um 1620, als sich in einigen Studienjahren über 200 Studenten inskribierten, ihren Höchststand. Die Frequenz fiel jedoch in der Universitätszeit kontinuierlich ab. Nach 1740 lag die Zahl der Neueinschreibungen regelmäßig unter 50 pro Jahr.

Der Altdorfer Lehrkörper umfasste in der Regel etwas über 15 ordentliche Professoren; allerdings fehlen für die Zeit nach 1623 noch detaillierte Forschungen. ${ }^{7}$ Die Theologische Fakultät verfügte zumeist über drei, die Juristische über vier, die Medizinische über drei Lehrstühle. An der Philosophischen Fakultät waren drei bis acht Ordinarien tätig. ${ }^{8}$ An allen Fakultäten unterrichteten zeitweise Privatdozenten und Extraordinarii, die jedoch im Folgenden ebenso wie die Sprach- und Exerzitienmeister unberücksichtigt bleiben.

Insgesamt lassen sich zwischen 1575 und 1807 an der Nürnberger Hochschule in Altdorf 171 Berufungen auf ordentliche Professuren nachweisen. Die Berufungen verteilen sich auf die einzelnen Fakultäten wie folgt: Theologie 45, Jurisprudenz 43, Medizin 27 und Philosophie 56. Zu Professoren ernannt wurden 163 Gelehrte. Ihre Biografien werden im Folgenden sowohl in der Gesamtheit als auch nach Zeitabschnitten und Fakultäten differenziert untersucht. Dabei muss ich mich selbstredend auf zentrale biografische Aspekte konzentrieren. Eine erschöpfende Analyse erforderte eine monografische Darstellung.

5 Eine moderne Gesamtdarstellung der Universitätsgeschichte fehlt; vgl. Horst Claus RecKTENwALD, Aufstieg und Niedergang der Universität Altdorf, in: ders. (Hg.), Gelehrte der Universität Altdorf, Nürnberg 1966, S. 11-49 (wiederabgedruckt in: Zeitschrift für Bayerische Landesgeschichte 30, 1967, S. 242-263); Hanns Christof Brennecke - Dirk Niefanger - Werner Wilhelm Schnabel (Hgg.), Akademie und Universität Altdorf. Studien zur Hochschulgeschichte Nürnbergs, Köln-Weimar-Wien 2011; H. MARTI - K. MARTI-WeissenBACH, Nürnbergs Hochschule.

6 Vgl. Franz Eulenburg, Die Frequenz der deutschen Universitäten von ihrer Gründung bis zur Gegenwart, Leipzig 1904 (ND Berlin 1994), S. 85, 88, 100-103, 153, 162-165; daneben: Artur KrEINER, Die jährlichen Neueinschreibungen an Gymnasium, Academie und Universität Altdorf von 1575-1809, Mitteilungen des Vereins für Geschichte der Stadt Nürnberg 37, 1940, S. 340-345.

7 Für die Zeit von 1575 bis 1623 vgl. Wolfgang MäHrLe, Academia Norica. Wissenschaft und Bildung an der Nürnberger Hohen Schule in Altdorf (1575-1623), Stuttgart 2000.

8 H. C. Recktenwald, Aufstieg, S. 40-41. 


\section{Geografische und soziale Herkunft}

\section{a) Geografische Herkunft}

Die Analyse der Herkunftsorte (Geburtsorte) der Altdorfer Hochschullehrer ergibt das in Grafik 1 erkennbare Bild.

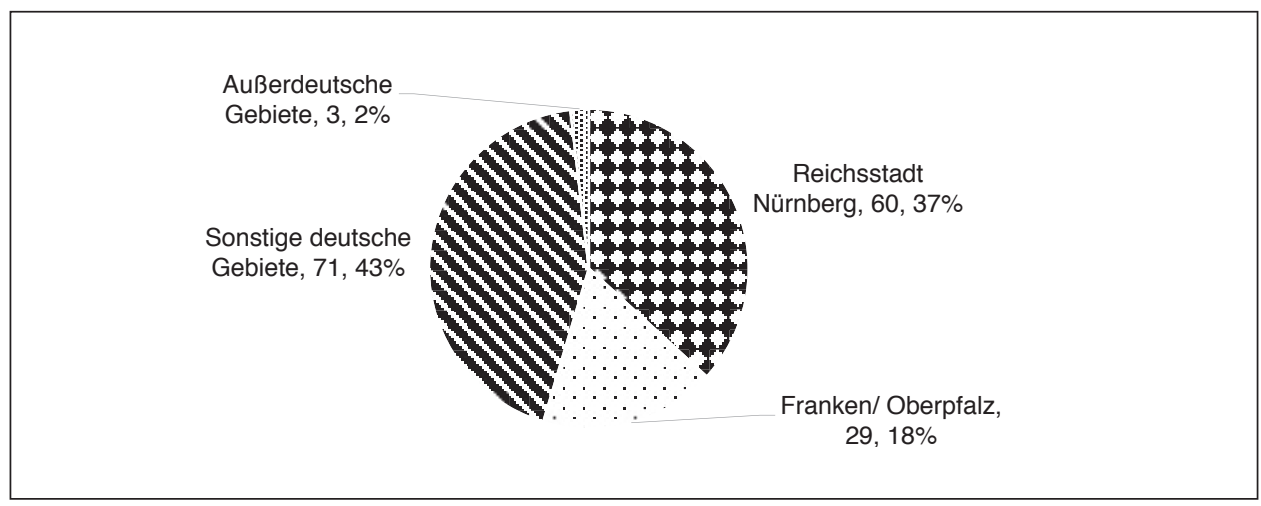

Grafik 1: Regionale Herkunft der Altdorfen Professoren 1575-1809

Demnach stammten von den insgesamt 163 auf Lehrstühle in Altdorf berufenen Gelehrten insgesamt 60 aus der Reichsstadt Nürnberg bzw. ihrem Landgebiet. ${ }^{9}$ Dies entspricht einem Anteil von 37 Prozent. 29 Professoren (= 18 Prozent) kamen aus der näheren geografischen Umgebung Nürnbergs, 71 (= 43 Prozent) aus weiter entfernt liegenden deutschen Territorien oder Städten einschließlich der bis 1648 noch formal zum Heiligen Römischen Reich gehörenden schweizerischen und niederländischen Gebiete. ${ }^{10}$ Lediglich drei Professoren wurden außerhalb des deutschen Kulturraums geboren. ${ }^{11}$

Vergleicht man den Anteil der „Landeskinder“ in Altdorf mit demjenigen an anderen mittelgroßen bzw. kleineren protestantischen Universitäten in der Frühen Neuzeit, so ist er als durchschnittlich einzustufen. Forschungsergebnisse, die einen direkten Vergleich ermöglichen, liegen seit den Studien von Matthias Asche insbesondere für den Zeitraum von 1650 bis 1799 vor. ${ }^{12}$ Berücksichtigt man lediglich diese Zeitspanne, so steigt der Anteil der Landeskinder an allen neu berufenen Professoren in Altdorf auf 44 Prozent. ${ }^{13}$ Dieser Wert liegt deutlich unter den Vergleichswerten, die für die Universitäten Rostock (57 Prozent) und Rinteln (51 Prozent) ermittelt werden konnten. ${ }^{14}$ Erheblich geringer war der Anteil der

9 Davon stammten 42 Professoren aus Nürnberg, 15 aus Altdorf, zwei aus Hersbruck und einer aus Lauf.

10 Die Berufung von insgesamt vier Schweizern und Niederländern auf Altdorfer Lehrstühle datiert ausschließlich in die Zeit vor 1648 .

11 Hugo Donellus (Berufung 1588), Scipio Gentilis (1590), Daniel Wilhelm Moller (1674).

12 M. Asche, Biographische Profile, S. 239 (Tabelle III).

13 Nicht berücksichtigt wurden demzufolge die vier Berufungen an die Universität Altdorf, die in den zehn Jahren zwischen 1799 und 1809 erfolgten. Zu Beginn des 19. Jahrhunderts wurden durchgehend Gelehrte nach Altdorf berufen, die von außerhalb des fränkischen Raums bzw. der Oberpfalz stammten.

14 Auch an der Universität Königsberg war der Anteil der Landeskinder - zumindest im von Bernhart Jähnig untersuchten 17. Jahrhundert - deutlich höher als in Altdorf, vgl. B. JäHNIG, Königsberger Universitätsprofessoren, 
Landeskinder in Helmstedt (35 Prozent), Heidelberg (27 Prozent), Kiel (26 Prozent) und Erlangen (23 Prozent). ${ }^{15}$ Doch sind die letztgenannten drei Universitäten aufgrund ihrer konfessionellen Ausrichtung bzw. als Neugründungen der Zeit nach 1650 mit Altdorf nur bedingt vergleichbar.

Der relative Anteil der Landeskinder an den neu berufenen Professoren ist an der Altorfina in der zweiten Hälfte des 17. Jahrhunderts mit 54 Prozent am höchsten, zwischen 1700 und 1809 sinkt er wieder auf 38 Prozent (Grafik 2). Dieser Befund weicht von dem an anderen Universitäten ab. Dort wurden die Spitzenwerte vielfach erst zu Beginn des 18. Jahrhunderts erreicht. ${ }^{16}$

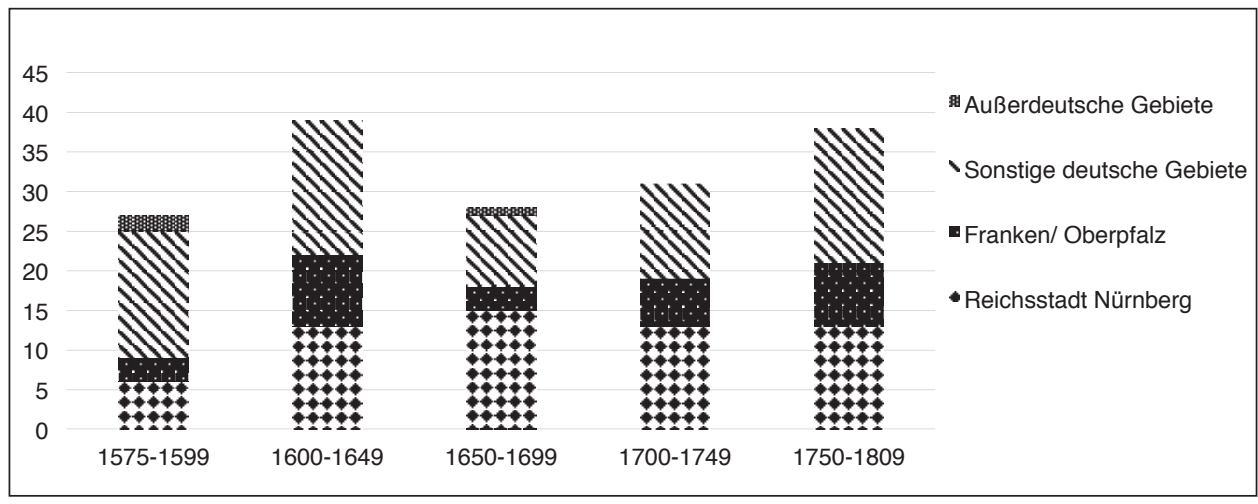

Grafik 2: Regionale Herkunft der Altdorfen Professoren 1575-1809: Chronologische Analyse

Interessant ist die regionale Herkunft der in der Grafik unter „Sonstige deutsche Gebiete" zusammengefassten Professorengruppe. Die Analyse zeigt, dass von den 71 Gelehrten, die dieser Gruppe zugehören, allein 30, das heißt fast die Hälfte, aus dem Vogtland, aus Thüringen oder aus Sachsen einschließlich der unmittelbar angrenzenden Gebiete des Vogtlands und des Erzgebirges stammten.. Dies weist auf eine enge Verflechtung der Universität Altdorf mit der mitteldeutschen Bildungslandschaft hin. Auf diesen Punkt wird noch zurückzukommen sein.

Schlüsselt man die geografische Herkunft der Professoren nach Fakultäten auf, so zeigen sich deutliche Unterschiede (Grafik 3). Der Anteil der Landeskinder war an der Nürnberger Hochschule vor allem an der Philosophischen und an der Theologischen Fakultät mit Werten von über 40 Prozent sehr hoch. Demgegenüber stammten die nach Altdorf berufenen Medizinprofessoren nur zu 22 Prozent aus Nürnberg bzw. dem reichsstädtischen Landgebiet. Der Anteil der Landeskinder an den Juristen lag bei 30 Prozent. Eine Erklärung

S. 342-346. Dasselbe dürfte für die bisher noch nicht systematisch untersuchte Universität Straßburg gelten; vgl. August Schricker, Zur Geschichte der Universität Straßburg. Festschrift zur Eröffnung der Universität Straßburg am 1. Mai 1872, Straßburg 1872, S. 49.

15 Nach S. Piotrowski, Sozialgeschichte, S. 124-126, betrug in Kiel im Zeitraum von 1665 bis 1815 der Anteil der Landeskinder an allen Professoren unter Einschluss von außerordentlichen Professoren und Privatdozenten 34,7 Prozent.

16 M. Asche, Biographische Profile, S. 207-208, 239 (Tabelle III). In Kiel war der Anteil der Landeskinder ebenfalls in der zweiten Hälfte des 17. Jahrhunderts am höchsten; vgl. S. Piotrowski, Sozialgeschichte, S. 126. 
für diese Unterschiede zu finden, fällt nicht leicht, auch deswegen, weil sich an anderen Hochschulen zum Teil völlig abweichende Verhältnisse feststellen lassen. ${ }^{17}$ Möglicherweise waren die Nürnberger Schulpolitiker aus Gründen des spezifischen Profils und des Renommees ihrer Hochschule eher bereit, angesehene Juristen oder Mediziner von anderen Universitäten - und damit auch aus anderen Regionen - nach Altdorf zu holen als Dozenten der Theologischen oder der Philosophischen Fakultät. Bei der Berufung von Theologen hat der konfessionelle Aspekt eine wichtige Rolle gespielt, was unter Umständen Landeskinder begünstigen konnte. ${ }^{18}$

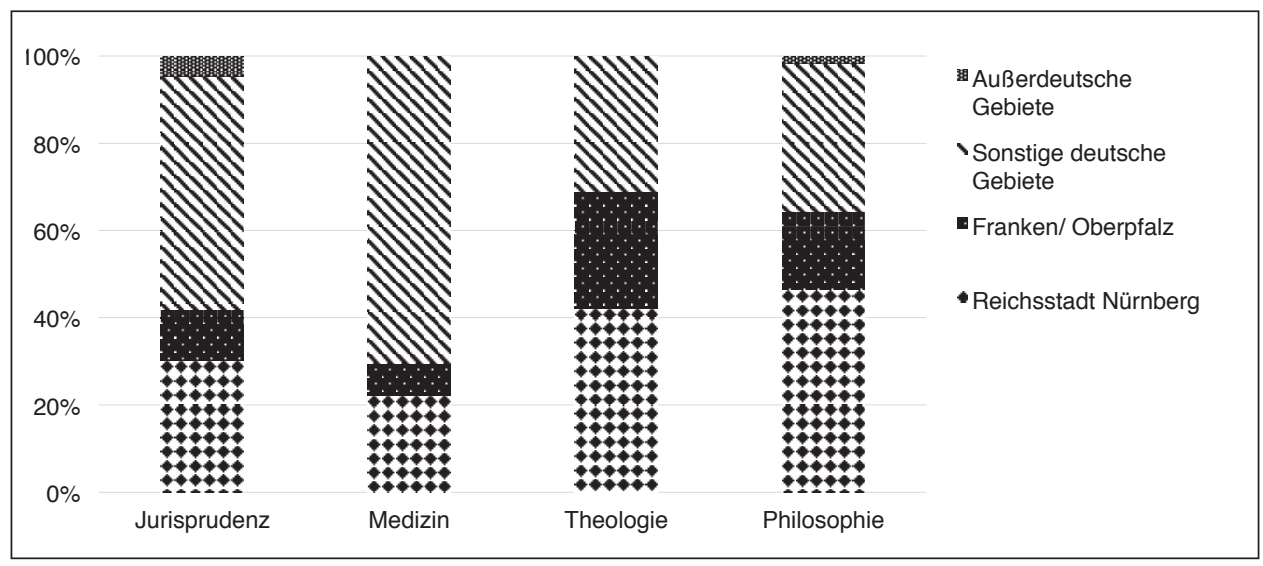

Grafik 3: Regionale Herkunft der Altdorfen Professoren 1575-1809: Fakultätsbezogene Analyse

Die Forschungen von Bernhard Homa ermöglichen es, den in Altdorf feststellbaren Anteil der Landeskinder an den Professoren der Philosophischen Fakultät im Zeitraum zwischen 1650 und 1749 mit den Gegebenheiten an anderen Universitäten zu vergleichen. ${ }^{19}$ Die Analyse ergibt für die Nürnberger Hochschule einen Wert von 65 Prozent. Dieser ist für mitteleuropäische Verhältnisse relativ hoch. Er wird jedoch an einigen Hochschulen noch übertroffen, so insbesondere an der von Homa eingehend untersuchten Universität Tübingen (85 Prozent).

17 Vgl. bes. M. Asche, Biographische Profile, S. 240 (Tabelle IV); B. JÄHNIG, Königsberger Universitätsprofessoren, S. 342-346; S. Piotrowski, Sozialgeschichte, S. 131-134.

18 Dieser Aspekt ist für die gesamte Zeitspanne von 1575 bis 1809 noch nicht systematisch erforscht, vgl. aber Klaus LeDER, Universität Altdorf. Zur Theologie der Aufklärung in Franken. Die Theologische Fakultät in Altdorf 1750-1809, Nürnberg 1965. Zum Kontext vgl. Ernst RiEgG, Eigenwille und Pragmatismus: Der Konflikt um die Norma Doctrinae in der Reichsstadt Nürnberg, in: Rudolf Schlögl (Hg.), Interaktion und Herrschaft. Die Politik der frühneuzeitlichen Stadt, Konstanz 2004, S. 237-267; Wolfgang MäHrLE, Die kirchliche Elite der Reichsstadt Nürnberg im konfessionellen Zeitalter (1553-1648), in: Michael Diefenbacher - Olga Fejtová - Zdzisław Noga (Hgg.), Krakau - Nürnberg - Prag. Die Eliten der Städte im Mittelalter und in der Frühen Neuzeit. Herkunft, Nationalität, Mobilität, Mentalität, Praha 2016, S. 281-311.

19 B. Hoмa, Tübinger Philosophische Fakultät, S. 217-226. 


\section{b) Soziale Herkunft}

Nicht für alle Altdorfer Professoren lassen sich präzise Informationen zur familiären Herkunft ermitteln. Aus diesem Grund wurde der nachfolgenden Analyse zum sozialen Hintergrund der Lehrstuhlinhaber ein sehr grobmaschiges Raster zugrunde gelegt, das lediglich drei Gruppen unterscheidet: Akademikersöhne, das heißt vor allem Söhne von Pfarrern, Professoren, Lehrern, Juristen, höheren Verwaltungsbeamten und Ärzten (Gruppe 1), Angehörige der politischen Führungsschichten bzw. der ökonomisch und sozial führenden Familien (Gruppe 2) und Sonstige, das heißt einfache Kaufleute, Handwerker, Bauern, sozial Schwache (Gruppe 3). ${ }^{20}$

Insgesamt sind auf der Grundlage der vorliegenden Gelehrtenlexika und anderer Nachschlagewerke zu 151 der 163 Universitätsprofessoren Aussagen zur sozialen Herkunft möglich (= 93 Prozent). Von diesen 151 Personen stammen 79 (=52 Prozent) aus Akademikerfamilien, 21 (= 14 Prozent) gehörten den gesellschaftlichen Eliten an, 51 (= 34 Prozent) der Gruppe 3 der „Sonstigen“. Ein Vergleich dieser Werte mit denjenigen an anderen Hochschulen ist nur mit Einschränkungen möglich, da die Zuweisung von Gelehrten zu sozialen Gruppen entsprechend den jeweiligen Analysezielen der bisher vorliegenden Studien bzw. den vorhandenen Quellen variiert. Der Anteil der Akademikersöhne an der Professorenschaft war in Altdorf ähnlich hoch wie an der wenige Jahrzehnte zuvor gegründeten Universität Marburg. ${ }^{21}$ An den später entstandenen Universitäten in Kiel, Halle und Erlangen lehrten demgegenüber prozentual mehr Professoren, die aus Akademikerfamilien stammten. ${ }^{22}$

Von den 79 Altdorfer Professoren aus Akademikerfamilien kamen 39, das heißt knapp 50 Prozent, aus Pfarrhaushalten. 14 (= 18 Prozent der Akademikersöhne) waren Kinder von Professoren, davon elf von Lehrstuhlinhabern an der Altorfina. ${ }^{23}$ Der Anteil der Söhne Altdorfer Professoren unter den Ordinarien an der reichsstädtisch-nürnbergischen Hochschule war damit mit weniger als 7 Prozent vergleichsweise niedrig. ${ }^{24}$

Untersucht man die soziale Herkunft der Altdorfer Professoren im Zeitablauf, so zeigt sich bis in die zweite Hälfte des 17. Jahrhunderts ein deutliches Ansteigen der Berufung von Akademikersöhnen (Grafik 4). Die Bedeutung dieser sozialen Gruppe bleibt dann im 18. Jahrhundert nahezu konstant. Bei der Entwicklung der anderen beiden Gruppen fällt vor allem der hohe Anteil an Angehörigen der Führungsschichten bei den Gelehrten auf, die im ausgehenden 16. und im frühen 18. Jahrhundert auf Altdorfer Lehrstühle berufen wurden.

20 Zur Problematik der sozialen Kategorisierung vgl. zuletzt ebd., bes. S. 226-231.

21 H. Niebuhr, Zur Sozialgeschichte, S. 44-80, bes. S. 46-50.

22 S. Piotrowski, Sozialgeschichte, S. 135-145; J. Schopferer, Sozialgeschichte, S. 98-116; O. Willett, Sozialgeschichte, S. 91-95.

23 Bei den drei Lehrstuhlinhabern, die Söhne eines nicht in Altdorf amtierenden Professors waren, handelte es sich um die Söhne des aus Nürnberg stammenden Jenaer und Hallenser Theologieprofessors Johann Wilhelm Baier (1647-1695).

24 M. AschE, Biographische Profile, S. 216, 241 (Tabelle V); für Königsberg vgl. B. JÄHNIG, Königsberger Universitätsprofessoren, S. 346-351; zu Kiel vgl. S. Piotrowski, Sozialgeschichte, S. 143; zu Halle vgl. J. SCHOPFERER, Sozialgeschichte, S. 103. 


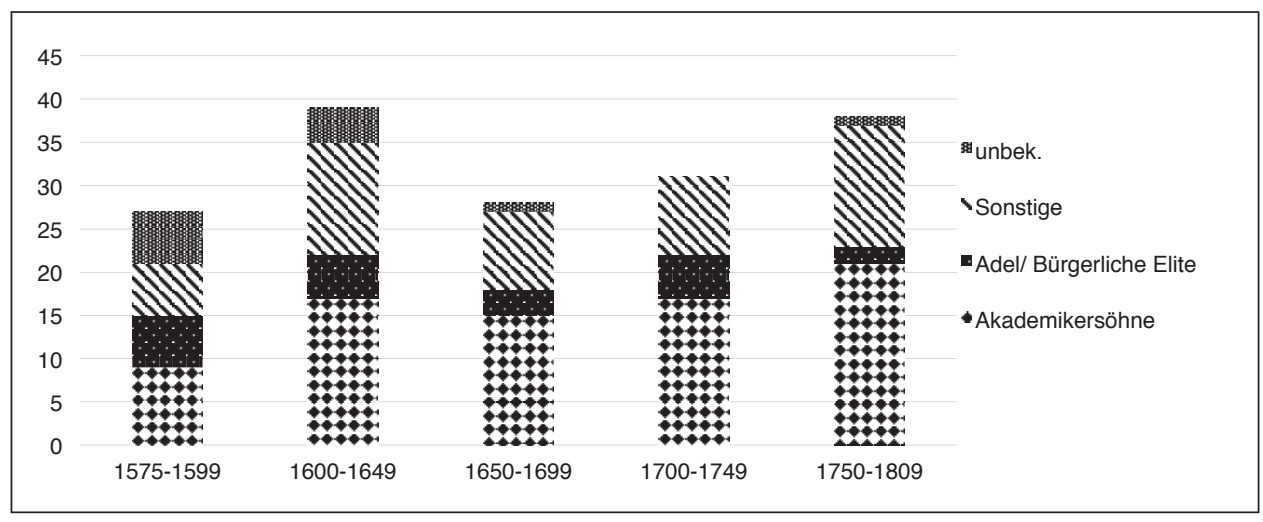

Grafik 4: Sociale Herkunft der Altdorfen Professoren 1575-1809: Chronologische Analyse

Die fakultätsbezogene Analyse bringt keine größeren Überraschungen: Angehörige sozialer Führungsschichten haben, wenn sie ein Professorenamt in Altdorf übernahmen, zumeist als Rechtsprofessoren gewirkt. Der Anteil der Akademikersöhne war besonders bei den Altdorfer Medizinern mit 59 Prozent sehr hoch. Die Philosophische und vor allem die Theologische Fakultät waren demgegenüber offener für Gelehrte ohne akademischen Familienhintergrund. ${ }^{25}$

\section{Bildungsweg}

Bei der Analyse des Bildungswegs der Altdorfer Professoren konzentriere ich mich auf das Hochschulstudium. Die Auswertung der personenbezogenen Daten ergibt, dass die Lehrstuhlinhaber der Altorfina bis zu acht Universitäten besucht haben. Im Durchschnitt studierten sie an 2,14 Hochschulen. Die Professoren der höheren Fakultäten besuchten häufiger mehrere Universitäten als die Lehrstuhlinhaber der Philosophischen Fakultät.

Analysiert man, auf welche Universitäten sich die Inskriptionen der späteren Altdorfer Professoren verteilen, so zeigt sich die überragende Bedeutung der Altorfina selbst, aber auch der mitteldeutschen Universitäten in Wittenberg, Leipzig und vor allem Jena für die akademische Bildung und Prägung der Gelehrten (Grafik 5). Auf die reichsstädtisch-nürnbergische Hochschule entfiel mehr als ein Viertel der insgesamt 346 feststellbaren Immatrikulationen; 93 der 163 späteren Altdorfer Lehrstuhlinhaber (= 57 Prozent) studierten dort. ${ }^{26}$ Dieser Wert liegt deutlich über dem Anteil der Landeskinder an allen Ordinarien.

25 Vgl. bezogen auf die Philosophischen Fakultäten B. HomA, Tübinger Philosophische Fakultät, S. $236-237$ (mit weiteren Nachweisen).

26 Der Anteil war andernorts noch deutlich höher. In Helmstedt lag er im 17. Jahrhundert bei 80 Prozent, in Halle im 18. Jahrhundert bei 76 Prozent; vgl. M. Asche, Helmstedter Professorenprofile, S. 114-115; Jens BRuning, Innovation in Forschung und Lehre. Die Philosophische Fakultät der Universität Helmstedt in der Frühaufklärung 1680-1740, Wiesbaden 2012, hier S. 100-101; J. SchOPFERER, Sozialgeschichte, S. 140. Auch in Königsberg scheint der Anteil an „Hausberufungen“ im 17. Jahrhundert höher als in Altdorf gewesen zu sein; vgl. B. JÄHNIG, Königsberger Universitätsprofessoren, S. 352-354. Niedriger war die Bedeutung Marburgs für die Ausbildung der dortigen Professoren. Lediglich 46 Prozent der Lehrstuhlinhaber hatten an der hessischen 
Den drei genannten mitteldeutschen Universitäten sowie der 1694 gegründeten Universität Halle lassen sich etwa 37 Prozent aller Inskriptionen Altdorfer Professoren zuordnen. 98 der 163 untersuchten Gelehrten haben an mindestens einer dieser vier Hochschulen studiert (= 60 Prozent). Dieser Befund belegt wiederum die enge Anbindung der Nürnberger Hochschule an die mitteldeutsche Bildungslandschaft. ${ }^{27}$ Die restlichen deutschen Universitäten sowie die ausländischen Bildungseinrichtungen spielten für den Bildungsgang der Altdorfer Lehrstuhlinhaber eine nachgeordnete Rolle. Erkennbar ist, dass sich die Zahl der von den Professoren besuchten Universitäten im 17. und 18. Jahrhundert reduziert.

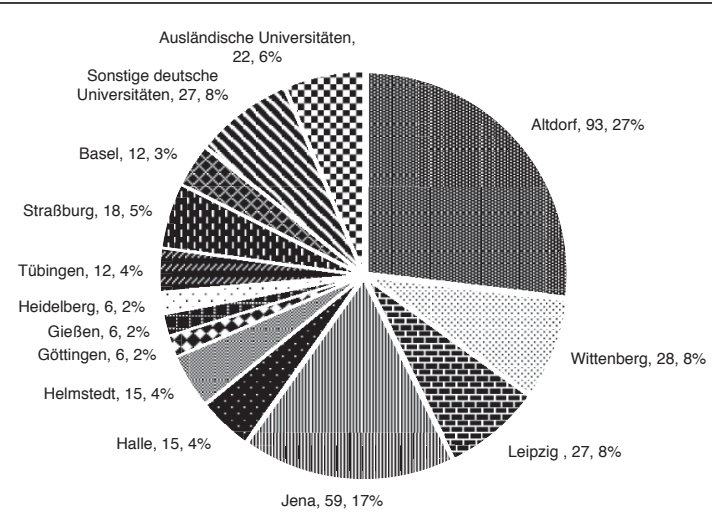

Grafik 5: Immatrikulationen Altdorfen Professoren an deutschen und ausländischen Universitäten

Sieht man von der Gründungsphase der Bildungseinrichtung ab, gab es während der über 230jährigen Geschichte der Altorfina zwei Hochschulen, die stets eine hohe Bedeutung für die Ausbildung der dort tätigen Professoren aufwiesen: die nürnbergische Lehranstalt selbst sowie die thüringische Universität Jena (Grafik 6). Andere Universitäten waren für die akademische Ausbildung der Altdorfer Professoren lediglich zeitweise wichtig. Dies gilt für die Universität Wittenberg, an der eine größere Zahl derjenigen Ordinarien studiert hatte, die bis zum Dreißigjährigen Krieg an die Altorfina berufen wurden; ebenso für die Universität Helmstedt, die im 17. Jahrhundert eine häufig besuchte Bildungseinrichtung war; für die Universität Leipzig, die die Ausbildungsstätte vieler im frühen 18. Jahrhundert nach Altdorf berufenen Gelehrten gewesen ist, sowie - mit Einschränkungen - auch für die späten Neugründungen in Halle und Göttingen. Hochschulen außerhalb des deutschen

Universität bereits studiert; vgl. H. NIEBUHR, Zur Sozialgeschichte, S. 101. Von den Kieler Professoren hatten etwa 37 Prozent an ihrer späteren Wirkungsstätte studiert; vgl. S. PIotrowsKi, Sozialgeschichte, S. 170.

27 Von einer lutherischen Bildungslandschaft „Franken-Schwaben“ kann aus der Perspektive dieses Aufsatzes nicht gesprochen werden, vgl. M. Asche, Biographische Profile, S. 231, 243 (Tabelle VII). Zur Frage der Bildungslandschaften in Süddeutschland vgl. Wolfgang MäHrLe, Straßburg als Vorbild. Das akademische Gymnasium Johannes Sturms und das evangelische höhere Bildungswesen in Süddeutschland (1540-1620), Historisches Jahrbuch 133, 2013, S. 167-224, und DERs., Süddeutsche Reichsstädter an der Universität Straßburg (1621-1793). Chronologie des Hochschulbesuchs, Bildungsziele, städtische und regionale Profile, in: Hanspeter Marti - Robert Seidel (Hgg.), Die Universität Straßburg zwischen Späthumanismus und Französischer Revolution, Wien-Köln-Weimar 2018, S. 379-462, hier bes. S. 459-462 (jeweils mit weiterer Literatur). 
Sprachraums wurden vor allem von den im ausgehenden 16. Jahrhundert nach Altdorf Berufenen besucht, in deutlich geringerer Zahl von denjenigen, die im 17. Jahrhundert einen Ruf an die Nürnberger Universität erhielten. Die Altdorfer Professoren des 18. Jahrhunderts studierten - von zwei Ausnahmen abgesehen - ausschließlich an deutschen Universitäten. ${ }^{28}$

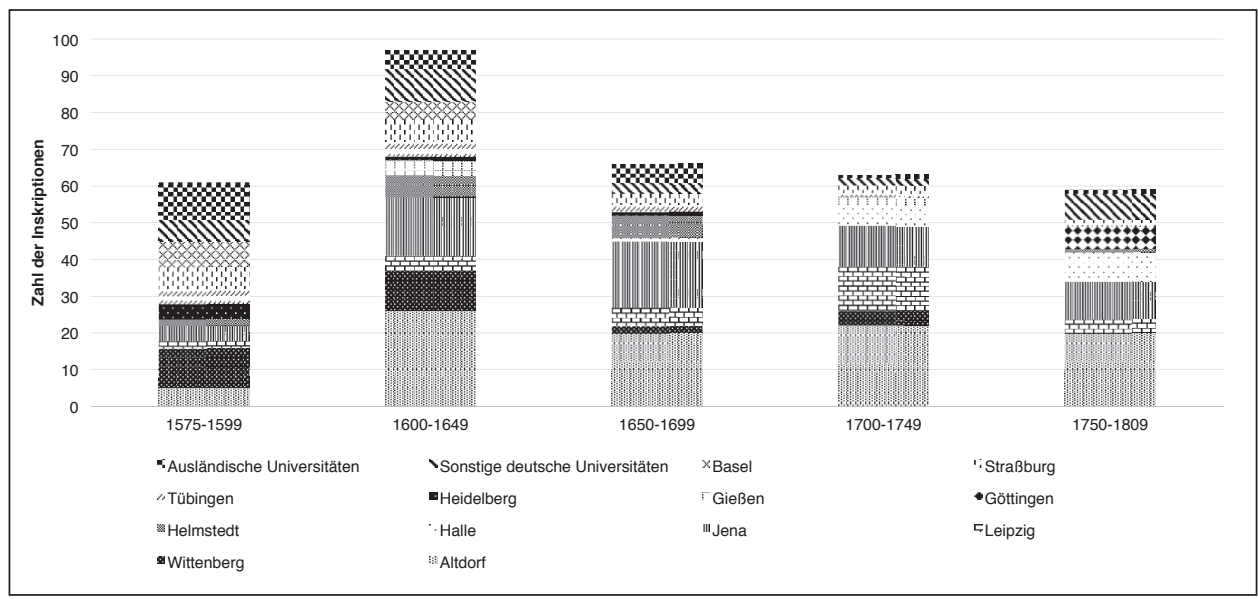

Grafik 6: Universitätsbesuch der Altdorfen Professoren: Chronologische Analyse

Der Hochschulbesuch der Altdorfer Gelehrten war selbstverständlich auch von ihren Berufszielen bestimmt. Dies zeigt eine Aufschlüsselung der frequentierten Bildungseinrichtungen nach der späteren Fakultätszugehörigkeit an der reichsstädtisch-nürnbergischen Hochschule. Grafik 7 verdeutlicht, dass an der Altorfina vor allem viele Professoren der Philosophischen Fakultät bereits studiert hatten. ${ }^{29}$ Beim Besuch anderer Hochschulen spiegeln sich die Lehrprofile dieser Bildungsinstitutionen. So war die Universität Jena vor allem eine wichtige Ausbildungsstätte der Altdorfer Theologen. An der Universität Wittenberg hatten viele Gelehrte studiert, die im späten 16. bzw. im frühen 17. Jahrhundert auf Philosophie- und Theologieprofessuren der Nürnberger Hohen Schule berufen wurden. Universitäten im Ausland, vor allem in Italien, aber auch in Frankreich wurden fast ausschließlich von späteren Altdorfer Medizinern und Juristen besucht.

Von wenigen Ausnahmen abgesehen, haben die Altdorfer Professoren ihr Studium mit einer Graduierung abgeschlossen. Während die späteren Rechts- und Medizinprofessoren in der Regel den Doktortitel erwarben, waren die Gelehrten, die auf Lehrstühle der Philosophischen oder Theologischen Fakultät berufen wurden, üblicherweise zum Magister promoviert worden. Die für die Übernahme einer theologischen Professur erforderliche Doktorpromotion erfolgte in vielen Fällen erst nach dem Dienstantritt in der Nürnberger Landstadt.

Untersucht man, wo die Altdorfer Professoren ihre akademischen Grade erworben haben, so zeigt sich, dass ein enger Zusammenhang zwischen dem Promotionsort und

28 Hochschulen im Ausland besuchten Lorenz Heister und Ludwig Philipp Wittwer. Die protestantische Universität Straßburg wurde auch für das 18. Jahrhundert zu den Hochschulen des deutschen Kulturraums gerechnet.

29 In Halle war vor allem an der Medizinischen Fakultät der Anteil von Professoren mit Studium in Halle sehr hoch; vgl. J. Schopferer, Sozialgeschichte, S. 141-142. 


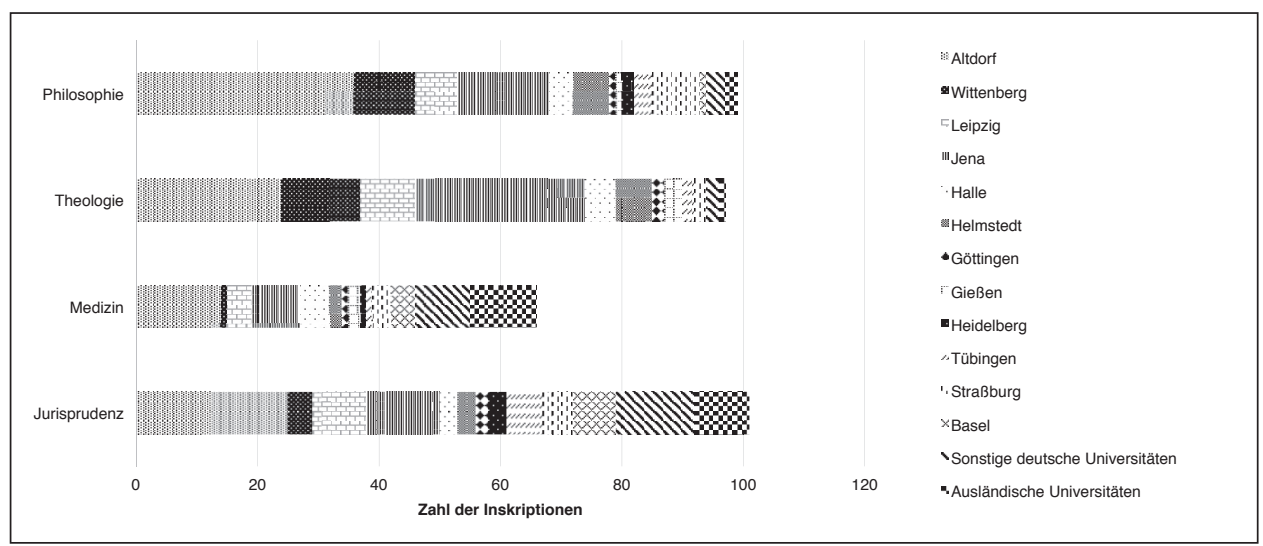

Grafik 7: Universitätsbesuch der Altdorfen Professoren: Fakultätsbezogene Analyse

der nachfolgenden Universitätskarriere bestand. ${ }^{30} 73$ der Altdorfer Professoren haben sich nachweislich an der Nürnberger Universität graduiert. Das entspricht einem Anteil von knapp 50 Prozent an allen 147 Professoren, die überhaupt einen akademischen Grad erwarben bzw. über deren Promotionsort(e) gesicherte Informationen vorliegen. ${ }^{31} 35$ der 73 Professoren erwarben in Altdorf den Doktortitel. Neun der 73 Professoren haben außer in Altdorf auch andernorts akademische Grade erworben - es handelt sich dabei mit einer Ausnahme jeweils um Professoren der Theologie, die nach ihrer Aufnahme ins Altdorfer Professorenkollegium zum Doktor der Theologie promovierten. Von den 64 Professoren, die sich ausschließlich an der Altorfina graduierten, waren 21 keine Nürnberger Landeskinder. Bei diesen 21 Personen wurde die Verbindung zur Altdorfer Hochschule also zumindest auch durch das mit einer Promotion abgeschlossene Studium hergestellt. Weitere Universitäten, an denen eine größere Zahl Altdorfer Professoren akademische Grade erwarb, waren die Hochschulen in Jena (23 Promovenden), Wittenberg (15) und Basel (9).

\section{Karrierewege vor Antritt der Altdorfer Professur}

Die Karrierewege der Altdorfer Professoren vor Antritt der Professur waren vergleichsweise vielfältig. Für 154 der 163 Ordinarien der Academia Norica liegen Informationen zu ihrer Tätigkeit zwischen Studienabschluss und der Berufung auf einen Lehrstuhl in der Nürnberger Landstadt vor. Von besonderer Bedeutung für die hier durchgeführte Analyse sind dabei die Dienstverpflichtungen, die im unmittelbaren zeitlichen Vorfeld der Berufung bestanden. Die diversen Karrierewege der Altdorfer Gelehrten lassen sich nur schwer kategorisieren, da die vorliegenden Informationen vielfach ungenau sind, mehrere Tätigkeiten parallel ausgeübt wurden oder sich verschiedene Aktivitäten in rascher Folge ablösten. Grob kann man vier Gruppen unterscheiden:

30 Auch an der Universität Königsberg zeigt sich der enge Zusammenhang zwischen Promotionsort und späterer Berufung; vgl. B. JÄHNIG, Königsberger Universitätsprofessoren, S. 354-357.

31 In Kiel lag dieser Wert bei knapp 36 Prozent; vgl. S. PIotrowski, Sozialgeschichte, S. 182. 
Die erste Gruppe umfasst Gelehrte, die zum Zeitpunkt der Berufung bereits Lehrstuhlinhaber an einer anderen Hochschule waren. Diese Gruppe ist in Altdorf mit lediglich 17 Personen sehr klein. Hinzu kommt, dass von den 17 Gelehrten vier unmittelbar vor ihrer Berufung aus konfessionellen Gründen ihre Stellung verloren hatten, sie also gar kein Professorenamt ausübten, als sie den Ruf an die Altorfina erhielten.

Zur zweiten Gruppe sind alle Personen zu rechnen, die sich zum Zeitpunkt der Berufung an einer Universität aufhielten und dort Lehrtätigkeiten wahrnahmen, jedoch keine ordentliche Professur bekleideten. Diese Gruppe umfasst etwa 40 Personen. Sie setzt sich zusammen aus Privatdozenten und außerordentlichen Professoren. Auch die jungen Hochschulabsolventen, die in Altdorf als Inspektoren der Stipendiaten wirkten, bevor sie auf einen Lehrstuhl berufen wurden, sind hierzu zu rechnen. Die Stipendiatenbetreuung war in Nürnberg mit einer Exspektanz auf einen Lehrstuhl verbunden.

Als dritte Gruppe lassen sich diejenigen Gelehrten abgrenzen, die zum Zeitpunkt ihrer Berufung nach Altdorf einer berufspraktischen Tätigkeit nachgingen. Es handelt sich mit etwa 80 Personen um die mit Abstand größte Gruppe. Unter den praktisch tätigen Akademikern findet sich jeweils eine signifikante Zahl an Ärzten, Pfarrern, Lehrern und Juristen bzw. Verwaltungsbeamten. Viele von ihnen, aber keinesfalls alle, standen in reichsstädtisch-nürnbergischen Kirchen-, Schul- oder Verwaltungsdiensten.

Schließlich schaffte eine wiederum sehr kleine Gruppe von etwa 15 Personen ohne längere Zwischentätigkeit den Sprung vom Studenten zum Professor. Ein Großteil dieser Gelehrten wurde im ausgehenden 16. und im 17. Jahrhundert auf eine Altdorfer Lehrkanzel berufen.

Vergleicht man die Altdorfer Verhältnisse mit denen an der vor wenigen Jahren von Julia Schopferer eingehend untersuchten Universität Halle, so fällt vor allem auf, dass der Anteil der Professoren, die vor ihrer Berufung auf einen Lehrstuhl einen praktischen Beruf ausübten, in Altdorf wesentlich größer als an der brandenburg-preußischen Hochschule gewesen ist. ${ }^{32}$ Belief er sich an der Altorfina auf etwa 52 Prozent, so betrug er in Halle nur etwa 28 Prozent. Ähnliche Karrierewege der Professoren wie in Altdorf konnte hingegen Swantje Piotrowski an der Universität Kiel nachweisen. ${ }^{33}$

\section{4. Übernahme und Ausübung des Professorenamts}

Die Altdorfer Professoren übernahmen ihr Amt im Alter von durchschnittlich 34,3 Jahren (Erstberufung auf eine Altdorfer Professur). ${ }^{34}$ Die Altersspanne der Neuberufenen war sehr groß. Sie reichte von 23 Jahren (Daniel Schwenter) bis 61 Jahren (Hugo Donellus, Jacob Tyde). Der Altersdurchschnitt der Neuberufenen war im ausgehenden 16. Jahrhundert mit 38,1 Jahren etwas höher als in den folgenden gut zwei Jahrhunderten. Dies liegt daran, dass in den Gründungs- und Aufbaujahren der Academia Norica verschiedentlich erfahrende (und damit ältere) Gelehrte nach Altdorf geholt wurden. Zwischen 1600 und 1809 pendelte

32 J. Schopferer, Sozialgeschichte, S. 151-180. Zu Erlangen vgl. O. Willett, Sozialgeschichte, S. 148-171.

33 S. Piotrowski, Sozialgeschichte, S. 184-204.

34 Zur Berufung von Gelehrten an Universitäten vgl. bes. William CLARK, Academic Charisma and the Origins of the Research University, Chicago 2006, hier S. 239-296, sowie den Sammelband Chr. HeSSE - R. Chr. SchwINGEs (Hgg.), Professorinnen und Professoren gewinnen. 
der Altersdurchschnitt der Neuberufenen - bezogen auf drei 50-Jahres-Zeiträume und einen 59-Jahres-Zeitraum - in dem schmalen Band zwischen 32,7 und 34,2 Jahren. Diese Werte sind, soweit erkennbar, für frühneuzeitliche Universitäten in Mitteleuropa nicht untypisch. ${ }^{35}$

Eine fakultätsbezogene Analyse des Lebensalters der Altdorfer Professoren bei Übernahme ihrer Stelle zeigt gewisse, wenngleich nicht allzu große Unterschiede. Die zum Zeitpunkt ihrer Berufung jüngsten Altdorfer Ordinarien waren die Mediziner mit durchschnittlich 31,5 Jahren, gefolgt von den Theologen (33,9 Jahre) und den Juristen (34,6 Jahre). Das höchste Eintrittsalter wiesen mit durchschnittlich 36 Jahren die Professoren der Philosophischen Fakultät auf. Vergleicht man das Eintrittsalter der Lehrstuhlinhaber an den Philosophischen Fakultäten der Universitäten Altdorf und Tübingen in den Jahren 1650 bis 1749 bzw. 1652 bis 1752, so zeigt sich lediglich eine geringe Abweichung von gut einem Jahr (Altdorf: 33,9 Jahre; Tübingen: 35,0 Jahre). ${ }^{36}$

Die Amtszeit der Altdorfer Professoren betrug durchschnittlich 19,3 Jahre. Während der verschiedenen Phasen der Hochschulgeschichte unterlag sie erheblichen Veränderungen. Die Verweildauer der im ausgehenden 16. Jahrhundert berufenen Professoren im Amt betrug lediglich 14,3 Jahre. In den folgenden 150 Jahren stieg dieser Wert - wieder bezogen auf 50-Jahres-Zeitabschnitte - über 20,5 (1600-1649) und 22,4 (1650-1699) Jahre auf 25,3 Jahre (1700-1749) deutlich an. Zwischen 1750 und 1809 ist dann ein rapider Rückgang der Amtszeiten auf 13,9 Jahre festzustellen. Die kurzen Amtszeiten im ausgehenden 16. Jahrhundert lassen sich dadurch erklären, dass zum einen in dieser Periode der Hochschulgeschichte, wie erwähnt, vielfach ältere Universitätslehrer für Altdorf gewonnen wurden. Zum anderen sind sie aber auch Ausfluss der energischen - und zum Teil aktivistischen - Schulpolitik der Nürnberger Scholarchen beim Aufbau der Hochschule, die zu Fehlbesetzungen von Lehrstühlen und zum frühzeitigen Abschied von Gelehrten führte. ${ }^{37}$ Der Hauptgrund für die kurzen Amtszeiten im ausgehenden 18. bzw. im frühen 19. Jahrhundert ist darin zu sehen, dass durch die Schließung der Universität nach dem Übergang der Reichsstadt Nürnberg an Bayern mehrere Universitätskarrieren - zumindest in Altdorf - abgebrochen wurden. Daneben amtierten einige Universitätslehrer nur kurz als ordentliche Professoren, waren jedoch zuvor lange Zeit, zum Teil mehrere Jahrzehnte, als Extraordinarii tätig gewesen. ${ }^{38}$

Analysiert man die Amtszeiten fakultätsbezogen, so sind keine sehr signifikanten Unterschiede feststellbar. ${ }^{39} \mathrm{Am}$ niedrigsten ist die durchschnittliche Verweildauer im Amt bei den Theologieprofessoren (17,5 Jahre), am längsten bei den Medizinern (21,9 Jahre). Vergleicht man wiederum die Amtszeiten an den Philosophischen Fakultäten der Universitäten Altdorf und Tübingen in den Zeiträumen zwischen 1650 und 1749 bzw. zwischen 1652 und

35 Zu Königsberg vgl. B. JÄHNIG, Königsberger Universitätsprofessoren, S. 361-365. Zu Erlangen vgl. O. WILLetT, Sozialgeschichte, S. 182-184. Zu Kiel vgl. S. Piotrowski, Sozialgeschichte, S. 221-222. Zu Tübingen vgl. den folgenden Absatz.

36 Zahlen nach B. НомA, Tübinger Philosophische Fakultät, S. 307.

37 Gelehrte, die Altdorf sehr rasch wieder verließen, waren Christian Francken (Lehrtätigkeit 1580), Gottlieb Mader (1583-1585), Matthias Indenius (1588-1590), Jacob Tetensius (1593-1594) und Christoph Coler (1598-1600). Zum Kontext vgl. W. MäHrLe, Academia Norica, bes. S. 157-159.

38 Wolfgang Jäger (13 Jahre Extraordinarius/ 9 Jahre ordentlicher Professor), Georg Christoph Schwarz (23 Jahre Extraordinarius/ 3 Jahre ordentlicher Professor).

39 Anders stellten sich die Verhältnisse an der Universität Kiel dar; dort war die Amtszeit vor allem der Juristen sehr kurz; vgl. S. PІотRowsкi, Sozialgeschichte, S. 260. 
1752, so fällt die deutlich längere Amtszeit der Altdorfer Professoren ins Auge. ${ }^{40}$ Während die Professoren der Philosophischen Fakultät in der Nürnberger Landstadt durchschnittlich 26 Jahre amtieren, wirkten die Tübinger in den einzelnen Professionen mit Ausnahme der Physiker bzw. Mathematiker lediglich zwischen 4,6 und 14,4 Jahren. Diese Divergenz begründet sich vor allem dadurch, dass in Tübingen die Inhaber von Lehrstühlen der Philosophischen Fakultät häufiger in eine der höheren Fakultäten aufrückten. ${ }^{41}$ In Altdorf waren die Möglichkeiten zum Avancement in die Theologische, die Juristische oder die Medizinische Fakultät extrem beschränkt. Im gesamten Zeitraum von 1575 bis 1809 schafften nur sechs Professoren der Philosophischen Fakultät diesen Karrieresprung. Fünf erlangten eine theologische, einer eine juristische Professur. ${ }^{42}$

Eine berufliche Veränderung war unter diesen Umständen für die Altdorfer Professoren der Philosophischen Fakultät nur innerhalb des eigenen Fachbereichs möglich. Immer wieder kam es vor, dass Gelehrte den Lehrstuhl wechselten oder neue Lehraufgaben zusätzlich übernahmen. An den höheren Fakultäten, vor allem bei den Theologen und Juristen, bestand eine Hierarchie in der Wertigkeit und Besoldung der Lehrstühle. Der Aufstieg erfolgte üblicherweise nach dem Anciennitätsprinzip.

Die Amtszeit der Altdorfer Professoren endete im Regelfall durch Tod, durch Wegberufung an eine andere Hochschule oder durch Übertritt in städtische oder landesherrliche Dienste (Grafik 8). Die überwältigende Mehrheit der Ordinarien übte ihr Amt bis zum Tod aus (59 Prozent). ${ }^{43}$ Am höchsten war der Anteil der im Amt Verstorbenen mit über 70 Prozent an der Philosophischen Fakultät. Dies bestätigt, dass eine Tätigkeit in diesem Fachbereich in Altdorf nur sehr geringe Karriereperspektiven bot. Einen Ruf an eine andere Universität nahmen 18 Altdorfer Hochschullehrer an (11 Prozent). 13 weitere erhielten Rufe, die sie aber ablehnten. Allein 15 der tatsächlich erfolgten Wegberufungen datieren ins 18. Jahrhundert; hierin ist ein Beleg dafür zu sehen, dass die Professuren in Altdorf in dieser Zeit im Vergleich zu anderen Universitäten wenig Renommee besaßen und vor allem sehr schlecht besoldet waren. Die Berufungen an andere Hochschulen verteilten sich in etwa zu gleichen Teilen auf Lehrstuhlinhaber aller Altdorfer Fakultäten. 23 Professoren der Altorfina wechselten zwischen 1575 und 1809 in städtische oder landesherrliche Dienste (13 Prozent). Darunter befanden sich allein 13 Theologen, die zumeist den Pfarrdienst in der Nürnberger Kirche oder in einem benachbarten Territorium der Lehrtätigkeit an der Altdorfer Universität vorzogen. Wenn sie in Nürnberg blieben, übernahmen die bisherigen Ordinarien häufig eine Pfarrei im reichsstädtischen Landgebiet. In kirchliche Führungspositionen der Reichsstadt Nürnberg gelangte auf direktem Weg keiner der Altdorfer Theologieprofessoren. ${ }^{44}$

40 B. Hома, Tübinger Philosophische Fakultät, S. 318.

41 Ebd., S. 320.

42 Wechsel in die Theologische Fakultät: Johann Wilhelm Baier (1709), Johann Conrad Dürr (1657), Jacob Wilhelm Feuerlein (1730), Theodoricus (Dietrich) Hackspan (1654), Johann Leonhard Schwäger (1666); Wechsel in die Juristische Fakultät: Johannes Kobius (1638).

43 Der Anteil der im Amt Verstorbenen an allen Professoren scheint in Königsberg noch höher gewesen zu sein; vgl. B. JäHNIG, Königsberger Universitätsprofessoren, S. 368-371. In Halle betrug der entsprechende Wert 66 Prozent, wobei vor allem die Theologen den Lehrstuhl bis zum Tod bekleideten; vgl. J. SCHOPFERER, Sozialgeschichte, S. 244-252. In Erlangen verstarben im 18. Jahrhundert 53 Prozent der Professoren im Amt, in Kiel 43 Prozent; vgl. O. Willett, Sozialgeschichte, S. 202-224, S. Piotrowski, Sozialgeschichte, S. 273.

44 Vgl. hierzu auch W. MährLe, Kirchliche Elite. 


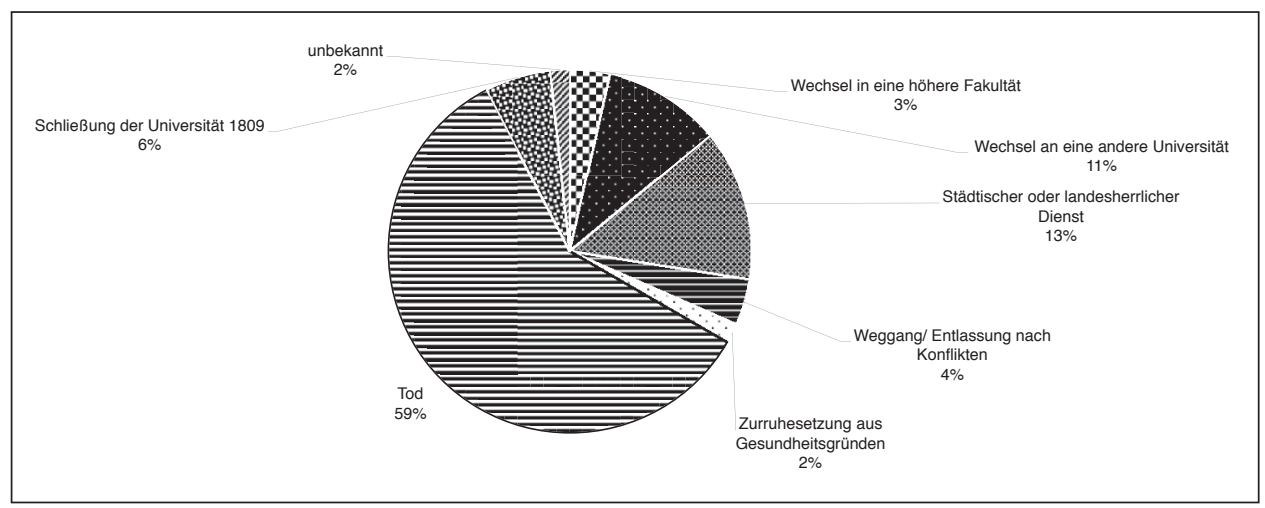

Grafik 8: Ausscheiden der Altdorfen Professoren aus dem Amt

\section{Altdorf - eine frühneuzeitliche „Familienuniversität“?}

In der jüngeren Vergangenheit ist in der Forschung wiederholt herausgestellt worden, dass die frühneuzeitlichen protestantischen Universitäten sozialgeschichtlich als „,Familienuniversitäten“ zu verstehen seien. ${ }^{45}$ Für zahlreiche Hochschulen (z. B. Gießen, Helmstedt, Kiel, Marburg, Rinteln, Rostock, Tübingen) lässt sich nachweisen, dass die dort wirkenden Gelehrten und ihre Familien vielfach miteinander verschwägert waren und sich demzufolge an diesen Bildungseinrichtungen im Verlauf mehrerer Generationen regelrechte Familienverbände bildeten, die einen beherrschenden Einfluss auf das akademische Leben, insbesondere auch auf die Neuberufungen von Professoren, ausübten. ${ }^{46}$ Erkennbar wurden

45 Vgl. bes. Peter MoRaw, Aspekte und Dimensionen älterer deutscher Universitätsgeschichte, in: ders. - Volker Press (Hgg.), Academia Gissensis. Beiträge zur älteren Gießener Universitätsgeschichte, Marburg 1982 , S. 1-45 (wiederabgedruckt in: DERS., Gesammelte Beiträge zur deutschen und europäischen Universitätsgeschichte, Strukturen - Personen - Entwicklungen, Leiden-Boston 2008, S. 3-54), hier S. 39-42; Matthias Asche, Über den Nutzen von Landesuniversitäten in der Frühen Neuzeit. Leistung und Grenzen der protestantischen „Familienuniversität“, in: Peter Herde - Anton Schindling (Hgg.), Universität Würzburg und Wissenschaft in der Neuzeit. Beiträge zur Bildungsgeschichte. Gewidmet Peter Baumgart anlässlich seines 65. Geburtstages, Würzburg 1998, S. 133-149; J. KüMMERLE, Wissenschaft und Verwandtschaft; DERS., „Absinkendes Niveau, fehlende Kritik und geringe Leistung “? Familienuniversitäten und Universitätsfamilien im Alten Reich, in: Daniela Siebe (Hg.), „Orte der Gelehrtheit“. Personen, Prozesse und Reformen an protestantischen Universitäten des Alten Reiches, Stuttgart 2008, S. 143-157; Heide WundER, ,,Die Professorin “ und die Professorentöchter - Ein Beitrag zur Sozialgeschichte des Professorenstandes in der Frühen Neuzeit, in: Horst Carl - Friedrich Lenger (Hgg.), Universalität in der Provinz. Die vormoderne Landesuniversität Gießen zwischen korporativer Autonomie, staatlicher Abhängigkeit und gelehrten Lebenswelten, Darmstadt 2009, S. 233-271.

46 P. Moraw, Aspekte, S. 50; DERs., Kleine Geschichte der Universität Gießen 1607-1982, Gießen 1982, S. 43-48; H. Niebuhr, Zur Sozialgeschichte, S. 122-145; Gerhard Schormann, Academia Ernestina. Die schaumburgische Universität zu Rinteln an der Weser (1610/21-1810), Marburg 1982, S. 198-207; M. Asche, Helmstedter Professorenprofile; DERS., Biographische Profile, S. 210-222; J. BRunING, Innovation, S. 100; Gustav KoHFELDT, Rostocker Professoren und Studenten im 18. Jahrhundert. Schilderungen nach den Akten und zeitgenössischen Berichten. Zur 500-Jahrfeier der Universität Rostock, Rostock 1919; B. HoмA, Tübinger Philosophische Fakultät, bes. S. 249, 355-366. Nur in Teilaspekten untersucht wurde das Professorenkollegium der Universität Greifswald, vgl. Dirk Alvermann, Die frühneuzeitliche „Familienuniversität“ im Spiegel der Greifswalder Professorenporträts, in: ders. - Birgit Dahlenburg, Greifswalder Köpfe. Gelehrenporträts und Lebensbilder des 16.-18. Jahrhunderts aus der pommerschen Landesuniversität, Rostock 2006, S. 23-30, sowie N. JöRN, Herkunft (jeweils mit weiterer Literatur). 
darüber hinaus Verflechtungen der dominierenden Geschlechter mit den Eliten im jeweiligen Trägerterritorium. ${ }^{47}$

Freilich waren verwandtschaftliche Verbindungen innerhalb der Professorenschaft nicht für alle frühneuzeitlichen protestantischen Universitäten in gleicher Weise prägend. ${ }^{48}$ Beispielsweise haben Studien zu den Universitäten Erlangen und Halle erkennen lassen, dass es dort nicht zur Ausbildung von einflussreichen Gelehrtendynastien gekommen ist. ${ }^{49}$ Vor diesem Hintergrund ist Julia Schopferer zuzustimmen, dass die bisherige wissenschaftliche Verwendung des Begriffs „Familienuniversität“ bisweilen zu undifferenziert und vielfach auch methodisch zu unreflektiert ist. ${ }^{50}$ Vorgeschlagen wird daher, den Begriff der „Familienuniversität“ auf diejenigen Hochschulen zu beschränken, in denen sich zum einen extensive Verwandtschaftsbeziehungen der Professoren nachweisen lassen und an denen sich zum anderen Gelehrtendynastien herausbilden konnten, die das akademische Leben dominierten.

Studien zur verwandtschaftlichen Verflechtung der an der Nürnberger Hochschule in Altdorf wirkenden Professoren sind auf der Grundlage des aktuellen Wissensstandes nicht vollumfänglich möglich. Die älteren Gelehrtenlexika, die die Grundlage für solche Analysen bilden müssen - einschlägig ist vor allem das erwähnte Lexikon von Will-Nopitsch -, halten die Familienverhältnisse der Altdorfer Gelehrten in weiten Teilen, aber nicht umfassend fest. Nahe Verwandtschaftsverhältnisse zwischen Professoren, das heißt neben Vater-Sohn-Beziehungen insbesondere Einheiraten von Gelehrten in Professorenfamilien, wurden - soweit erkennbar - vor allem von Georg Andreas Will zuverlässig registriert. ${ }^{51}$ Hingegen fehlen häufig Angaben zum Zeitpunkt der Eheschließung von Professoren, so dass die möglichen Auswirkungen der Verehelichung auf die Karriere eines Akademikers nicht immer adäquat eingeschätzt werden können.

Wertet man die zur Verfügung stehenden Daten aus, so lässt sich für 65 der 163 Altdorfer Professoren ein Verwandtschaftsverhältnis zu einem anderen Professor an der Academia

47 Dieser Aspekt wurde inzwischen für mehrere Universitäten bzw. Territorien erforscht. Aus universitätsgeschichtlicher Perspektive vgl. bes. Th. Sснмотz, Leipziger Professorenfamilien, bes. S. 27-32, 480-483; J. Schopferer, Sozialgeschichte, S. 289-345, sowie S. PIotrowski, Sozialgeschichte, S. 287-316. Aus territorialgeschichtlicher Perspektive vgl. etwa. Luise SCHORN-SchüTtE, Evangelische Geistlichkeit in der Frühneuzeit. Deren Anteil an der Entfaltung frühmoderner Staatlichkeit und Gesellschaft. Dargestellt am Beispiel des Fürstentums Braunschweig-Wolfenbüttel, der Landgrafschaft Hessen-Kassel und der Stadt Braunschweig, Gütersloh 1996.

48 Dies wird beispielsweise durch die Aufstellungen von M. Asche, Biographische Profile, S. 241-242 (Tabellen V und VI) deutlich. Vgl. auch die Bemerkungen von Hans HaEring, Die Hohe Schule Herborn - ein „Familienverband"? Mitteilungsblatt des Geschichtsvereins Herborn e.V. 39, 1991, S. 94-104, hier S. 101.

49 O. Willett, Sozialgeschichte, S. 67; J. Schopferer, Sozialgeschichte, S. 270-284.

50 J. Schopferer, Sozialgeschichte, S. 270-284, bes. S. 279-280. Schopferer kritisiert zu Recht, dass vormoderne Hochschulen bisweilen als „Familienuniversitäten“ etikettiert werden, ohne dass systematische Forschungen zu den Professorenkollegien vorliegen. Ebenso ist Schopferer zuzustimmen, wenn sie moniert, dass sozialhistorisch ausgerichtete Studien häufig zu wenig nach der Funktion von Verwandtschaftsbeziehungen für den Universitätsbetrieb bzw. für die akademischen Karrieren der Gelehrten fragen. Vielfach erfolgte die Einheirat von Gelehrten in bestehende Professorenfamilien erst nach Erlangung eines Lehrstuhls, häufig auch erst nach dem Tod des jeweiligen Schwiegervaters.

51 In den von Christian Conrad Nopitsch herausgegebenen Folgebänden des „Nürnbergischen Gelehrten-Lexicons" sind die Familienverhältnisse des Öfteren nicht wiedergegeben. Sie lassen sich zum Teil durch den Rückgriff auf andere Nachschlagewerke ermitteln. 
Norica nachweisen (= 40 Prozent). ${ }^{52}$ Insgesamt elf Söhne Altdorfer Ordinarien erhielten einen Lehrstuhl an der reichsstädtisch-nürnbergischen Hochschule, ${ }^{33} 25$ Altdorfer Professoren (= 16 Prozent) waren Schwiegersöhne eines Lehrstuhlinhabers. Weitere Verwandtschaftsbeziehungen bestanden in Großvater-Enkel-Beziehungen (1), Onkel-Neffen-Beziehungen (2) oder entstanden durch die Berufung von Schwägern (1) bzw. Brüdern (drei Brüder Baier, zwei Brüder Spies).

Die Zahlen verdeutlichen, dass familiale Verbindungen konstitutiv für die Zusammensetzung des Altdorfer Professorenkollegiums gewesen sind. Die weitere Analyse zeigt jedoch auch sehr eindrücklich, dass die verwandtschaftliche Verflechtung an der Altorfina eher gering war. Untersucht man, mit wie vielen Kollegen die oben erwähnten 65 Lehrstuhlinhaber - generationenübergreifend - verwandt waren, so erhält man folgendes Ergebnis: Acht Professoren waren mit sieben Kollegen verwandt, sieben mit sechs, acht mit drei, 18 mit zwei Kollegen und 24 lediglich mit einem anderen Ordinarius. Dies belegt klar, dass sich in Altdorf keine weitläufigen Verwandtschaftsbeziehungen etablieren und sich insbesondere keine dominierenden Gelehrtendynastien ausbilden konnten. ${ }^{54}$ Ansätze hierzu sind lediglich in den Jahrzehnten um 1700 zu erkennen, als sich um den Mediziner Moritz Hoffmann sowie um die drei Brüder Baier zwei „Familienverbände“ bildeten, der insgesamt acht bzw. sieben Professoren angehörten. ${ }^{55}$ Aufgrund der spezifischen Chronologie der Verwandtschaftsverhältnisse bzw. der - zum Teil weit auseinanderliegenden - Amtszeiten der Ordinarien konnten die beiden Sippen jedoch nicht einmal für kürzere Zeit eine vorherrschende Stellung an der Altorfina erlangen. ${ }^{56}$

Analysiert man, welche Rolle verwandtschaftliche Verhältnisse bei der Neuberufung von Altdorfer Professoren spielten, erhält man ein ebenso bemerkenswertes Ergebnis. Es erweist sich, dass an der Academia Norica die Protektion durch Ordinarien in Berufungsverfahren keine hohe Bedeutung erlangte..$^{57}$ Von den insgesamt 65 Lehrstuhlinhabern, für die sich ein Verwandtschaftsverhältnis mit einem anderen Altdorfer Professor ermitteln lässt, haben nach den vorliegenden Informationen höchstens 21 (=13 Prozent aller Altdorfer Lehrstuhlinhaber) von der Fürsprache durch einen Familienangehörigen profitieren können bzw. mutmaßlich profitiert. ${ }^{58}$ Der bruchlose Übergang einer Professur auf einen

52 Die Altdorfer Verhältnisse sind diesbezüglich fast identisch mit denen in Rinteln. Dort lässt sich bei 68 von 171 Lehrkräften ein Verwandtschaftsverhältnis nachweisen. Doch war die Zahl der Professorensöhne (in Rinteln: 26) in Altdorf weitaus geringer; vgl. G. Schormann, Academia Ernestina, S. 200.

53 Dabei handelte es sich um neun Vater-Sohn-Beziehungen sowie eine Vater-Sohn-Enkel-Beziehung.

54 Der Altdorfer Befund ähnelt in diesem Punkt den Forschungsergebnissen von Julia Schopferer für die Universität Halle; vgl. J. SchOPfERER, Sozialgeschichte, S. 282-284.

55 Zum „Familienverband“ um den Mediziner Moritz Hoffmann zählten folgende Professoren: Georg Nößler, Moritz Hoffmann, Johann Moritz Hoffmann, Johann Gerhard Frauenburger, Johannes Fabricius und sein gleichnamiger Sohn, Johann Georg Fichtner und Balthasar Adam Werner. Mit den drei Brüdern Baier (Johann Wilhelm, Johann Jacob und Johann David) waren verwandt: Johann Ludwig Apin, Johann Albrecht und Wolfgang Albrecht Spies und Johann Bartholomäus Riederer.

56 Das Ergebnis einer geringen verwandtschaftlichen Verflechtung der Altdorfer Universitätsprofessoren geht konform mit meinen Forschungsergebnissen zur Nürnberger kirchlichen Elite während des konfessionellen Zeitalters. Auch die Nürnberger Geistlichen gelangten nur selten aufgrund verwandtschaftlicher Beziehungen in Leitungspositionen; vgl. W. MÄHRLE, Kirchliche Elite, S. 301, 309-310.

57 Vgl. hierzu die in Anmerkung 34 genannte Literatur sowie M. Asche, Biographische Profile, S. $223-236$.

58 Bei zwei der 21 Personen ist dies aufgrund der Quellenlage unsicher. 
Verwandten - quasi eine Art „Vererbung“ - lässt sich nur in vier Fällen nachweisen. ${ }^{59}$ Bei den anderen 44 Gelehrten bestand zum Zeitpunkt der Berufung (noch) kein Verwandtschaftsverhältnis (31 Personen) oder war der ältere Verwandte zum Zeitpunkt der Berufung bereits tot (13 Personen). Im letzteren Fall könnte das Verwandtschaftsverhältnis einen indirekten Einfluss auf die Einstellung ausgeübt haben.

Aufgrund der oben erwähnten Defizite der älteren Gelehrtenlexika ist nicht auszuschließen, dass die in diesem Aufsatz präsentierten Ergebnisse durch zukünftige personengeschichtliche Forschungen noch präzisiert bzw. gegebenenfalls auch in einzelnen Punkten modifiziert werden können. Mit einer grundlegenden Revision ist allerdings nicht zu rechnen. Legt man einer abschließenden Bewertung die oben skizzierte, eher restriktive Definition des Begriffs „Familienuniversität“ zugrunde, so kann Altdorf demnach nicht als eine solche, von familialen Strukturen geprägte Hochschule bezeichnet werden. Verwandtschaftliche Beziehungen der Professoren spielten dort zwar eine nicht unerhebliche Rolle, doch waren sie kein dominierendes Strukturmerkmal des Universitätsbetriebs. Es konnten sich in Altdorf insbesondere keine Professorendynastien ausbilden, die das akademische Leben nachhaltig bestimmten. Auch bei der Berufung von Professoren waren in der ganz überwiegenden Mehrzahl der Fälle andere Kriterien als das der Verwandtschaft $\mathrm{zu}$ amtierenden oder ehemaligen Altdorfer Ordinarien entscheidend. Dieser Befund ist insofern bemerkenswert, als die Altorfina im Unterschied zu den von Olaf Willett und Julia Schopferer untersuchten Universitäten in Erlangen und Halle bereits im 16. Jahrhundert gegründet wurde. Die Ausbildung dichter verwandtschaftlicher Netzwerke kann daher in der Nürnberger Landstadt nicht an einer zu kurzen Generationenfolge bis zur Aufhebung der Universität im Jahr 1809 gescheitert sein. ${ }^{60}$ Die zukünftige Forschung wird systematisch nach den Gründen für die spezifischen Altdorfer Gegebenheiten fragen müssen. Dabei wird nicht zuletzt die Rolle der reichsstädtisch-nürnbergischen Schulverwaltung in den Blick zu nehmen sein.

\section{WOLFGANG MÄHRLE}

\section{Raněnovověká „,rodinná univerzita“? Profesorské kolegium norimberské vysoké školy a univerzity Altdorf 1575-1809}

\section{RESUMÉ}

Přítomná studie zkoumá profesorské kolegium vysoké školy, resp. univerzity Altdorf po dobu jejího trvání 1575-1809, a to pod zorným úhlem kolektivní biografické metody. V Altdorfu přednášelo celkově 163 řádných profesorů. Obrazu známému z jiných raněnovověkých vysokých škol odpovídá skutečnost, že mnoho stolic na Altorfině obsadili přednášející, kteří z teritoria sídla školy, v případě Altdorfu tedy z říšského města Norimberku, pocházeli a/nebo na místě svého budoucího působení studovali. Výrazné jsou kromě toho úzké vazby altdorfských profesorů na středoněmecké univerzity v Halle, Jeně, Lipsku a Wittenbergu. Jako „rodinná univerzita“ nemůže

59 Georg König / Theodoricus (Dietrich) Hackspan (1654); Johann Paul Felwinger / Johann Jobst Felwinger (1671); Johann Paul Felwinger / Georg Paul Rötenbeck (1681); Johann Wilhelm Baier / Johann David Baier (1730).

60 M. Asche, Biographische Profile, S. 216, stellte fest, dass die Ausbildung der charakteristischen Strukturen einer Familienuniversität üblicherweise zwei bis drei Generationen in Anspruch nahm. 
být Altdorf označen. Rodinné vazby tam sice nehrály zanedbatelnou roli, přesto př́ibuzenské zasítování uvnitř profesorského kolegia bylo slabé a na škole se nemohly etablovat žádné učenecké rody.

(český překlad Blanka Zilynská)

Dr. Wolfgang Mährle

Landesarchiv Baden-Württemberg - Hauptstaatsarchiv Stuttgart

E-mail: wolfgang.maehrle@la-bw.de 